\title{
Validity of the New Lifestyles NL-1000 Accelerometer for Measuring Time Spent in Moderate-to-Vigorous Physical Activity in School Settings
}

\author{
David McMinn, David A. Rowe, Michelle Stark, and Louise Nicol \\ Department of Sport, Culture and the Arts, University of Strathclyde, Glasgow, Scotland
}

\begin{abstract}
Current interest in promoting physical activity in the school environment necessitates an inexpensive, accurate method of measuring physical activity in such settings. Additionally, it is recognized that physical activity must be of at least moderate intensity in order to yield substantial health benefits. The purpose of the study, therefore, was to determine the validity of the New Lifestyles NL-1000 (New Lifestyles, Inc., Lee's Summit, Missouri, USA) accelerometer for measuring moderate-to-vigorous physical activity in school settings, using the Actigraph GT1M (ActiGraph, Pensacola, Florida, USA) as the criterion. Data were collected during a cross-country run $(n=12)$, physical education $(n=18)$, and classroom-based physical activities $(n=42)$. Significant and meaningful intraclass correlations between methods were found, and NL-1000 estimates of moderate-to-vigorous physical activity were not meaningfully different from GT1M-estimated moderate-to-vigorous physical activity. The NL-1000 therefore shows promising validity evidence as an inexpensive, convenient method of measuring moderate-to-vigorous physical activity in school settings.
\end{abstract}

Key words: accelerometry, children, moderate-to-vigorous physical activity, physical education, measurement

\section{INTRODUCTION}

In adults, regular physical activity contributes to the prevention of several chronic diseases that are associated with a risk of premature death (United States Department of Health and Human Services, 1996; Warburton, Nicol, \& Bredin, 2006). Additionally, physical inactivity can increase the risk of life-threatening diseases and lead to premature mortality (Parliamentary Office of 
Science and Technology, 2001). Furthermore, physical activity in youth promotes healthy growth and development of the musculoskeletal and cardiorespiratory systems, and provides opportunities for social interaction, achievement, and mental well-being (Department of Health, Physical Activity, Health Improvement and Prevention, 2004).

School settings have been identified as key social establishments in which to promote physical activity, particularly through the medium of school clubs and physical education (PE) classes (Fairclough \& Stratton, 2006; Strong et al., 2005; Wechsler, Devereaux, Davis, \& Collins, 2000; Young et al., 2007). Valid methods of estimating physical activity in children and adolescents are critical to understanding the relationship between physical activity and chronic diseases and associated risk factors (Tudor-Locke, Williams, Reis, \& Pluto, 2004). Many studies have been conducted with the aim of providing an accurate measure of physical activity in children and adolescents. However, these studies have often highlighted the limitations of various measures when used in an adolescent population. Sirard and Pate (2001) concluded that an ideal measure of physical activity for use in children and adolescents remains elusive. Moreover, there is still a need for an accurate, practical, and affordable measure of physical activity that can be implemented within the school setting (Scruggs, 2007).

The New Lifestyles NL-1000 (New Lifestyles, Inc., Lee's Summit, Missouri, USA) is an innovative and affordable $(\approx \$ 50$ per unit $)$ motion sensor, based on a piezoelectric accelerometer mechanism. In addition to the standard function of recording steps, the NL-1000 has a function that has been labeled the Moderate-to-Vigorous Physical Activity ${ }^{\mathrm{TM}}$ Timer (MVPA $^{\mathrm{TM}}$ Timer). This function records the accumulated time spent in physical activity of at least moderate intensity; it has been suggested that activity of moderate intensity is more beneficial to health than light intensity activity (American College of Sports Medicine, 2000; United States Department for Health and Human Services, 1996). If validity evidence can be provided for the NL-1000 for measuring time spent in MVPA, it may prove useful for measuring health-promoting physical activity in school settings. Therefore, the purpose of this study was to provide validity evidence for the New Lifestyles NL-1000 for use as an accurate, practical, and affordable physical activity measurement tool in school settings, specifically for measuring MVPA.

\section{METHODS}

The study was designed to collect criterion-related validity evidence using an ActiGraph GT1M (ActiGraph, Pensacola, Florida, USA) accelerometer as the reference method. Data were collected under three different conditions, i.e., during a continuous cross-country run, during a PE lesson, and during classroom-based physical activity breaks. All procedures were approved by the ethics committee of the University of Strathclyde before data collection began. Parent and child informed consent was obtained for all participants.

\section{Instruments}

\section{ActiGraph GT1M}

The GT1M is a compact uni-axial accelerometer measuring $3.8 \times 3.7 \times 1.8 \mathrm{~cm}$ in size. In this study, the GT1Ms were drawn from a pool of 18 instruments and were worn on an elastic belt 
and positioned on the left hip. Piezoelectric transducers and microprocessors record accelerations that are converted to digital signals known as "counts." Counts are recorded at a rate of 30 Hertz and summed for a pre-selected epoch length, ranging from $1 \mathrm{sec}$ to $1 \mathrm{~min}$. Epoch lengths can be altered to suit research needs. For example, a 1-min epoch may be used for studies lasting a long period of time, e.g., a week, whereas a 1-sec epoch may be used in shorter studies, e.g., a PE lesson. Data produced by the GT1M are activity counts and steps. The sum of activity counts per epoch is linearly related to activity intensity; i.e., as activity intensity increases, so do activity counts (McClain, Sisson, Washington, Craig, \& Tudor- Locke, 2007b). Validated cutpoints can be used to determine activity intensities. The GT1Ms were initialized, and data were downloaded using Actilife ${ }^{\mathrm{TM}}$ data analysis software (version 3.2.2; ActiGraph, Pensacola, Florida, USA) in accordance with the manufacturer's guidelines. Initialization involves setting the time, date, epoch length, and file name. In this study, 1-sec epochs were used. Prior to the initializing the GT1M, the computer used to initialize the GT1M was time synchronized with a digital watch, allowing for accurate data screening.

\section{$N L-1000$}

The NL-1000 (New Lifestyles, Inc., Lee's Summit, MO) is an innovative piezoelectric pedometer measuring $6.4 \times 3.8 \times 2 \mathrm{~cm}$ in size that can be clipped onto a belt or waistband. In this study, NL-1000s were drawn from a pool of 18 instruments and worn alongside the GT1M on an elasticized waistband. The NL-1000 uses a piezoelectric mechanism that is similar to accelerometers, but it costs a fraction of the price. In addition to counting steps, the NL-1000 records time spent in MVPA. The NL-1000 MVPA $^{\mathrm{TM}}$ timer may be set to record at intensities above any one of nine discrete intensity levels, and it is delivered from the manufacturer set to record activity above 3.6 Metabolic Equivalents (METs) (Level 4). However, perhaps the most common definition of MVPA is activity that is at or above an intensity of 3 METs (American College of Sports Medicine, 2000). Therefore, the NL-1000 MVPA timers in this study were set to record activity above a threshold of 2.9 METs (Level 3). Steps and MVPA time are read from a digital display.

\section{PROCEDURES}

\section{Cross-Country Run Condition}

Twelve school children aged 10-13 years were recruited from a community comprehensive school in the west of Glasgow. The participants were part of a school running club that met each morning to run before the start of school. On the morning of the designated period of data collection, the aim of the study was explained to the participants, and verbal instructions were given on the correct placement and use of the activity monitors. All NL-1000s were reset to zero before data collection. After ensuring that all belts were being worn correctly, participants took part in their run, which was led by one of the school PE teachers. On returning from the run, participants laid their belts with attached monitors on a gym hall bench and NL-1000 steps and NL-1000 MVPA times were recorded. The start and finish times of the run were recorded using the previously synchronized digital watch to allow for accurate analysis of the GT1M data. 
GT1M data were downloaded and saved later the same day. Not all participants took part in the same run, due to children returning their consent forms at different times.

\section{PE Condition}

Eighteen children aged 10 and 11 years were selected from a community comprehensive school in the west of Glasgow to wear the activity monitors during a PE lesson. The lesson content was track and field activities, which were selected because they were intermittent in nature and, therefore, tested the validity of the NL-1000 over a wider variety of activity intensities than the cross-country run condition. Before the lesson began, the aims of the study were explained and participants were instructed on how to wear the activity monitors. Participants were lined up in front of the activity monitors and instructed to put their belts on simultaneously. The start time was recorded using the previously synchronized digital watch. After the PE lesson had finished, the finish time, NL-1000 steps, and NL-1000 MVPA times were recorded, and the GT1M data were downloaded and saved later the same day.

\section{Classroom Activity Breaks Condition}

Two grade-5 and two grade- 6 classes $(N=68)$ were recruited from a state school in the west of Scotland to be monitored while taking part in classroom activity breaks (CAB). The Class Moves! ${ }^{\circledR}$ (Fysio Educatief, Amsterdam) is a classroom-based program that engages children in short bouts $(\approx 5-15 \mathrm{~min})$ of physical activity with the aim of improving their work and concentration. The children had been taking part in The Class Moves! for several weeks prior to data collection; therefore, the usual class schedule was not changed. Data were collected over a twoweek period. Each class participated in three sessions of CAB. Participants were given a unique ID number that corresponded with a belt containing the two activity monitors. It was therefore possible to assign the same activity monitors to each participant for each of the activity sessions. Monitors were placed at participants' desks before each activity session; after all NL-1000s were reset to zero, participants were instructed to put on their belts. This time was recorded to ensure synchronization of the data. Belts were then checked to ensure that all monitors were positioned correctly. The classroom teacher then led the class through an activity session as normal. On each day, all four classes performed the same activity as each other, and a different activity was performed on the three days of data collection. At the end of the activity session, participants were instructed to remove their belts in unison and place them on their desks. Finish time, NL-1000 steps, and NL-1000 MVPA times were recorded, and GT1M data were downloaded and saved later the same day.

\section{Data Treatment}

\section{ActiGraph}

Data from each GT1M were downloaded onto a secure computer at the University of Strathclyde. When first downloaded, GT1M data were displayed in a data file (.dat) format. These files were saved as comma separated values (.csv) files and then converted into Microsoft Excel 
(Microsoft Corp., Redmond, Washington, USA) format ready for data screening. Initially, data from each participant were cleaned. This involved deleting data collected by the GT1M when the participant was not wearing the monitor, leaving data for activity time only. Time stamps created through the use of the synchronized digital watch were used to determine start and finish times of activity. The following age-specific MET prediction equation developed by Freedson, Sirard, Debold, Pate, Dowda, and Sallis (1997) was used to determine cutpoints to identify time spent in MVPA for each participant:

$$
\begin{aligned}
\text { METs }= & 2.757+(.0015 * \text { counts } / \mathrm{min})-(.08957 * \text { age }[\text { years }]) \\
& -(0.000038 * \text { counts } / \mathrm{min} * \text { age }[\text { years }]) .
\end{aligned}
$$

Using 3 METs as the threshold for MVPA, 1-sec cutpoints were calculated for ages 9-13 years (the MET prediction equation was developed using participants aged 6-18 and is therefore suitable for use with the participants in this study). One-min cutpoints of 906 to 1,399 counts/min were therefore converted to 1-sec cutpoints of 15 to 23 counts/sec (i.e., any observed count above the threshold was added to MVPA time). Specific age cutpoints were important because the association between accelerometer counts and energy expenditure differs between age groups. The COUNTIF macro function on Microsoft Excel was then used to identify cells containing data at or above this threshold, giving total time (sec) spent in MVPA. Total activity time (sec) and total step count were also calculated, using the SUM function in Excel. Although 3 METs has been used to denote the threshold for MVPA in children (e.g., Trost, Way, \& Okely, 2006), recent commentators have suggested that a higher threshold (4 METs) should be used to denote MVPA in children. Consequently, 4-MET cutpoints of 1,769 to 2,393 counts/min also were used (corresponding to 1-sec cutpoints of 29 to 39 counts/sec) in the current study. These two criterion measures are referred to as GT1M-3MET and GT1M-4MET throughout the rest of this manuscript.

Total activity time (sec), MVPA time (sec) using a 3-MET threshold and also using a 4-MET threshold, and total step count for each participant were entered into the master data sheet (Excel). After entering all data into the Excel master sheet, the data were subsequently imported to SPSS (v. 15.0; SPSS Inc., Chicago, Illinois, USA) for further analyses. Descriptive statistics and histograms were created to allow for data screening and the identification of outliers or individuals with missing data. Data were judged to be normal if skewness and kurtosis were within reasonable bounds $(<|2.0|)$ and if minimum and maximum values were realistically possible. There were no obvious outliers or individuals with missing data during the cross-country run condition (RUN). One participant from the PE condition was identified as having a relatively high GT1M MVPA score $(1,228 \mathrm{sec})$ in comparison to his/her NL-1000 MVPA score $(516 \mathrm{sec})$ and was judged to be a bivariate outlier. It was decided that data analysis would be run with this participant included and also with the participant excluded to determine the effect of the outlier data-point on the results. Under the CAB condition, data across the three $\mathrm{CAB}$ days were summed for each participant. One grade- 6 class missed a day of data collection due to a school trip, and these data were omitted from data analysis. Additionally, nine children from the other three classes also missed at least one day of data collection, and their data were omitted from data analysis. This left a working data file of 42 children under the CAB condition. 


\section{$N L-1000$}

Total steps and MVPA time for each participant under each condition were entered into the master data sheet. MVPA min and sec were converted into sec. Error scores (GT1M - NL-1000) were calculated for all three activities.

\section{Statistical Analysis}

Means, standard deviations, skewness, kurtosis, minimum, and maximum values were calculated for the NL-1000 and GT1M step data and time spent in MVPA. Mean differences were assessed using $t$-tests, and Cohen's $d$ (Cohen, 1969) gave an indication of effect size. Intraclass correlation coefficients (ICC) from a two-way ANOVA model, adjusted for a single instrument, with 95\% confidence interval were calculated for MVPA time for the NL-1000 and GT1M.

\section{RESULTS}

All MVPA data were relatively normally distributed (see Table 1). Skewness and kurtosis were within reasonable bounds $(<|2.0|)$, as were minimum and maximum values. GT1M steps under the PE condition were positively kurtotic (3.29).

TABLE 1

Descriptive Statistics for GT1M and NL-1000 Steps and MVPA Time

\begin{tabular}{|c|c|c|c|c|c|c|}
\hline Condition & Mean & $S D$ & Skewness & Kurtosis & Minimum & Maximum \\
\hline \multicolumn{7}{|l|}{$\operatorname{Run}(n=12)$} \\
\hline Time (sec) & $1,066.42$ & 236.03 & 0.44 & -0.22 & 769 & 1,537 \\
\hline NL-1000 steps & $2,956.50$ & 615.70 & 0.35 & -1.37 & 2,176 & 3,868 \\
\hline NL-1000 MVPA (sec) & 994.67 & 201.88 & 0.42 & -1.01 & 748 & 1,320 \\
\hline GT1M steps & $2,829.75$ & 626.94 & 0.34 & -1.29 & 2,067 & 3,757 \\
\hline GT1M 3-MET (sec) & 996.75 & 211.72 & 0.47 & -0.59 & 725 & 1,379 \\
\hline GT1M 4-MET (sec) & 968.75 & 208.61 & 0.34 & -0.92 & 687 & 1,315 \\
\hline \multicolumn{7}{|l|}{$\operatorname{PE}(n=18)$} \\
\hline Time (sec) & $3,818.00$ & & & & & \\
\hline NL-1000 steps & $2,353.56$ & 411.43 & 0.31 & -0.66 & 1,727 & 3,135 \\
\hline NL-1000 MVPA (sec) & 808.22 & 190.35 & 0.51 & -0.12 & 516 & 1,204 \\
\hline GT1M steps & $1,852.33$ & 301.63 & 1.47 & 3.29 & 1,505 & 2,739 \\
\hline GT1M 3-MET (sec) & $1,064.83$ & 147.03 & -0.26 & -0.61 & 802 & 1,325 \\
\hline GT1M 4-MET (sec) & 830.00 & 119.71 & 0.03 & 0.78 & 571 & 1,089 \\
\hline \multicolumn{7}{|l|}{$\mathrm{CAB}(n=42)$} \\
\hline Time (sec) & $1,015.86$ & 214.03 & 0.19 & -1.66 & 780 & 1,278 \\
\hline NL-1000 steps & $1,048.05$ & 259.33 & 0.32 & -0.32 & 606 & 1,676 \\
\hline NL-1000 MVPA (sec) & 331.74 & 100.65 & 0.64 & 0.01 & 176 & 612 \\
\hline GT1M steps & 721.26 & 199.30 & 0.25 & -0.92 & 404 & 1,148 \\
\hline GT1M 3-MET (sec) & 416.19 & 94.09 & 0.22 & -0.46 & 253 & 643 \\
\hline GT1M 4-MET (sec) & 349.93 & 81.25 & 0.39 & -0.12 & 205 & 568 \\
\hline
\end{tabular}


TABLE 2

Mean Comparison and Intraclass Correlation Coefficient for GT1M and NL-1000 MVPA Times

\begin{tabular}{|c|c|c|c|c|c|c|c|}
\hline Condition & $n$ & Mean difference & $F$ & $p$ & $d$ & $I C C$ & $\mathrm{CI}_{95}$ \\
\hline \multicolumn{8}{|l|}{ Run } \\
\hline NL-1000 vs. GT1M-3MET & 12 & 2.08 & 0.08 & .78 & 0.01 & 0.99 & $0.98-1.00$ \\
\hline NL-1000 vs. GT1M-4MET & 12 & -25.92 & 22.99 & $<.01$ & -0.13 & 1.00 & $0.99-1.00$ \\
\hline \multicolumn{8}{|l|}{ PE } \\
\hline NL-1000 vs. GT1M-3MET & 18 & 256.61 & 43.90 & $<.01$ & 1.52 & 0.53 & $0.10-0.80$ \\
\hline NL-1000 vs. GT1M-4MET & 18 & 21.78 & 0.29 & .60 & 0.14 & 0.42 & $0.00-0.74$ \\
\hline \multicolumn{8}{|l|}{$\mathrm{CAB}$} \\
\hline NL-1000 vs. GT1M-3MET & 42 & 84.45 & 169.41 & $<.01$ & 0.87 & 0.93 & $0.83-0.95$ \\
\hline NL-1000 vs. GT1M-4MET & 42 & 18.19 & 6.80 & .01 & 0.20 & 0.88 & $0.78-0.93$ \\
\hline \multicolumn{8}{|l|}{ PE (outlier removed) } \\
\hline NL-1000 vs. GT1M-3MET & 17 & 229.82 & 60.00 & $<.01$ & 1.41 & 0.72 & $0.39-0.89$ \\
\hline NL-1000 vs. GT1M-4MET & 17 & -1.94 & 0.00 & .96 & -0.01 & 0.57 & $0.14-0.82$ \\
\hline
\end{tabular}

Run

During the RUN condition, there was no significant mean difference between NL-1000 MVPA time and GT1M-3MET (mean difference $=2.08 \mathrm{sec}, p \geq .05$; see Table 2), but NL-1000 MVPA time was significantly higher than GT1M-4MET (mean difference $=-25.92 \mathrm{sec}, p<.05$ ). Cohen's $d$ indicated a trivial effect size for both comparisons, however $(d=.01$ and $d=-.13)$. There was a high correlation between NL-1000 MVPA and each of the two GT1M MVPA estimates $\left(\mathrm{ICC}=.99, \mathrm{CI}_{95}=.98-1.00\right.$, and $\mathrm{ICC}=1.00, \mathrm{CI}_{95}=.99-1.00$ for GT1M-3MET and GT1M-4MET, respectively).

PE

During the PE condition, the NL-1000 significantly underestimated MVPA time compared with GT1M-3MET (mean difference $=256.61 \mathrm{sec}, p<.05$ ) but was not significantly different from GT1M-4MET (mean difference $=21.78 \mathrm{sec}, p>.05$ ). Cohen's $d$ indicated a large effect size $(d=1.52)$ for the GT1M-3MET comparison but a trivial effect size $(d=.14)$ for the GT1M-4MET comparison. There was a weak correlation between NL-1000 MVPA and both GT1M estimates of MVPA ( ICC $=.53, \mathrm{CI}_{95}=.10-.80$, and $\mathrm{ICC}=.42, \mathrm{CI}_{95}=.00-.74$ with GT1M-3MET and GT1M-4MET, respectively). After removing the bivariate outlier, the NL-1000 again significantly underestimated GT1M-3MET (mean difference $=229.82 \mathrm{sec}, p<$ .05 ) and was not significantly different from GT1M-4MET (mean difference $=-1.94 \mathrm{sec}$, $p>$.05). Similarly, Cohen's $d$ indicated a large effect size $(d=1.41)$ for the GT1M-3MET comparison and a trivial effect size $(d=-0.01)$ compared to GT1M-4MET. The correlation between instruments improved when the outlier was removed, however $\left(\mathrm{ICC}=.72, \mathrm{CI}_{95}=.39-.89\right.$, and $\mathrm{ICC}=.57, \mathrm{CI}_{95}=.14-.82$ with GT1M-3MET and GT1M-4MET, respectively). 
During the CAB condition, the NL-1000 significantly underestimated time spent in MVPA compared to GT1M-3MET (mean difference $=84.45 \mathrm{sec}, p<.05$ ) and was also significantly lower than GT1M-4MET (mean difference $=18.19 \mathrm{sec}, p<.05$ ). While the GT1M-3MET comparison was a medium to large effect size $(d=.87)$, the comparison with GT1M-4MET was a trivial effect size $(d=.01)$. There was a moderately good correlation between NL-1000 MVPA and both GT1M MVPA estimates $\left(\mathrm{ICC}=.93, \mathrm{CI}_{95}=.83-.95\right.$, and $\mathrm{ICC}=.88, \mathrm{CI}_{95}=.78-.93$ with GT1M-3MET and GT1M-4MET, respectively).

\section{DISCUSSION}

The purpose of this study was to provide validity evidence for the New Lifestyles NL-1000 pedometer for use as an accurate, practical, and affordable measure of MVPA in school settings. The primary finding of this study was that estimates of MVPA time from the NL-1000 are, on average, similar to estimates derived from accelerometry using age-specific equations and a 4-MET threshold to define moderate intensity. This was true for a variety of school-based activities (cross-country run, PE, and classroom physical activity breaks). Other than during running, the NL-1000 underestimated accelerometry-derived MVPA with 3 METs used as the threshold. The absolute validity of the NL-1000 for estimating time spent in MVPA during school-based physical activity was therefore supported, if one accepts 4 METs as an appropriate MVPA threshold in children. Absolute validity refers to the ability of an instrument to measure accurately the correct score for a construct, in this case MVPA time (Sallis \& Saelens, 2000). This is important for situations such as reporting population values of MVPA or for determining the proportion of children who meet MVPA recommendations. During running, the choice of GT1M-derived MVPA threshold minimally affected the agreement with NL-1000 MVPA. The most likely explanation for this finding is that when running, children are usually considerably and continuously above the moderate intensity threshold, regardless of whether this is defined as 3 METs or 4 METs; thus, agreement between instruments is relatively easy to achieve. However, testing the NL-1000 under these conditions was a necessary prerequisite before testing it under more challenging conditions (i.e., where physical activity intensity varied and moderate intensity physical activity was intermittent). Activity during PE and classroom-based activity, on the other hand, is more intermittent and varied in nature; including light, moderate, and vigorous intensity activities. Therefore, any disparity in MVPA recording ability between instruments is more likely to be detected during these conditions. The results in the current study, therefore, are promising for use of the NL-1000 to determine MVPA in school-based physical activity.

The correlations between the NL-1000-derived MVPA and GT1M-derived MVPA were moderate to high under all conditions (although the removal of an outlier was necessary to achieve this in the PE condition), with one exception. The correlation with GT1M-4MET MVPA was fairly low. Other than the latter results, this supports the relative validity of the NL-1000 for measuring MVPA, i.e., its ability to put children in a similar order to the GT1M (Sallis \& Saelens, 2000). 
Previous studies using activity monitors with the same internal sensor as the NL-1000 have shown agreement with the ActiGraph accelerometer when measuring time spent in MVPA (McClain, Johnson, Brusseau, Washington, Tudor- Locke, \& Darst, 2007a; McClain et al., 2007b). These studies used different ActiGraph epoch lengths (15 sec and $30 \mathrm{sec}$ ) than the current study and different cutpoints and equations to determine MVPA thresholds. Children's physical activity patterns tend to be intermittent and varied, alternating between short bouts of only a few seconds of differing intensities (Bailey, Olson, Pepper, Porszasz, Barstow, \& Cooper, 1995). Because of this, the use of longer epochs tends to produce lower estimates of MVPA time compared to shorter epochs (Mahar, Smith, Rowe, DuBose, \& McCammon, 2008). This issue aside, McClain et al. (2007a) found that the NL-1000 set at the default MVPA threshold of Level 4 produced similar estimates of MVPA to the ActiGraph in eighth-grade PE. The ActiGraph was used in conjunction with a cutpoint of 3,000 counts/min corresponding to 4.6 METs, derived from the equation of Treuth et al. (2004) developed in eighth-grade girls. Similarly, McClain et al. (2007b) found agreement between NL-1000 and ActiGraph estimates of free-living MVPA in 10-year-old children over a day, when NL-1000 MVPA was measured using Level 5 and the ActiGraph was used in conjunction with cutpoints of 3,000 counts/min (Treuth et al., 2004) or 3,200 counts/min (Puyau, Adolph, Vohra, Zakeri, \& Butte, 2004). However, large differences were found between the NL-1000 MVPA at other settings and the Actigraph used with other cutpoints.

The results of the current study should be interpreted in the context of its limitations. Data were collected on convenience samples that were relatively small, particularly for the Run and PE conditions. The PE data were collected on only one type of activity (track and field), albeit purposively selected in order to represent activity of intermittent and varied intensity. Additionally, the choice of criterion (particularly the selection of accelerometer cutpoints) should be considered. Current debate surrounds the translation of accelerometer counts into estimated activity intensity and remains a contentious issue, particularly with regard to the correct cutpoints to be used in children. Consensus is lacking on which is most suitable (see, for example, Guinhouya \& Hubert, 2008; Sherar, Baxter-Jones, Esliger, \& Tremblay, 2008). Several validated moderate intensity activity cutpoints have been proposed for use in children, for example, ranging from 615 counts/min to 3,200 counts/min for the ActiGraph accelerometer (Corder, Ekeland, Steel, Wareham, \& Brage, 2008). These include the Freedson et al. (1997) age specific cutpoints used in the current study, Puyau et al. (2004; 3,200 counts/min), and Treuth et al. (2004; 3,000 counts/min). The variety of published cutpoints poses obvious difficulties for researchers trying to identify a suitable cutpoint and is certainly a limiting factor (Baquet, Stratton, Van Praagh, \& Berthoin, 2007). A further confounding factor is the choice of 3 METs or some other intensity to denote the moderate physical activity threshold in children. While a higher intensity (most notably 4 METs) has been proposed for children, there is some ambiguity regarding whether this refers to 4 "adult" METs (i.e., $4 \times 3.5 \mathrm{ml} / \mathrm{kg} / \mathrm{min}=14 \mathrm{ml} / \mathrm{kg} / \mathrm{min} \mathrm{O}_{2}$ ) or whether one MET refers to resting energy expenditure. Children's resting energy expenditure is higher than an "adult" MET_-for example, ranging in various studies of measured resting energy expenditure from $3.8 \mathrm{ml} / \mathrm{kg} / \mathrm{min}$ (Treuth et al., 2004) to $5.9 \mathrm{ml} / \mathrm{kg} / \mathrm{min}$ (Harrell, McMurray, Baggett, Pennell, Pearce, \& Bangdiwala, 2005). However, when divided by measured resting energy expenditure, the MET cost of most physical activities is similar to that of adults (Ridley \& Olds, 2008), implying that 3 METs (where 1 MET is defined as the child's resting energy expenditure) may be an appropriate intensity threshold for moderate intensity. Finally, the choice of activity 
threshold for the NL-1000 also should be considered. In the current study, Level 3 (2.9 METs) was selected. Because the NL-1000 accelerometer mechanism was validated during walking activity in adults (Kumahara et al., 2004), it is unclear whether this corresponds to the same intensity in children during mixed (ambulatory and non-ambulatory) activity.

Future research in this area should address some of the limitations just described. In particular, it is important that there is agreement on a defined method of calculating activity cutpoints for children to use with GT1M data. Additionally, the NL-1000 MVPA Timer could be tested during activities of known intensity, for example to determine at what walking speed the MVPA Timer starts to engage. It may also be useful to explore the validity of the NL-1000 in free living conditions or during a variety of activities such as games and sports. Finally, future NL-1000 validity studies should be conducted using larger samples in order to achieve greater statistical reliability.

Researchers seeking to measure MVPA in the school setting should consider the positive and negative features of the NL-1000 before selecting it over other instruments such as the GT1M accelerometer. Positive features of the NL-1000 include the digital display that allows steps and MVPA time to be read directly from the screen, meaning that no downloading or cleaning of data is required. This offers an attractive advantage over the GT1M device, which requires often laborious downloading and cleaning of data. Further positive features of the NL-1000 include the 7 -day memory function and the relatively inexpensive price. Negative features of the NL-1000 should also be highlighted. Although the digital display allows for ease of access to data, this feature may also contribute to participant reactivity, particularly in children, who often shake pedometers in order to increase their step score. In addition to this, open access to the buttons that reset the device could lead to participants resetting their device, whether intentionally or by accident, impacting the reliability of data gathered; these are negative features not associated with the GT1M device that has no display or buttons. Before a device is selected for use in research, these positive and negative features should be considered based on individual study requirements.

\section{CONCLUSION}

There is a need for an affordable activity monitor that can accurately measure time spent in MVPA in school settings. This would provide physical activity practitioners with a measurement tool that could be used in large groups to provide feedback on whether levels of activity intensity meet the minimum level for enhancing health. The current study provides promising validity evidence that the NL-1000 could be used for this purpose.

\section{ACKNOWLEDGMENTS}

The data were collected as part of the undergraduate dissertations of David McMinn, Michelle Stark, and Louise Nicol under the supervision of David Rowe. 


\section{REFERENCES}

American College of Sports Medicine. (2000). Guidelines for exercise testing and prescription (6th ed.). Philadelphia: Lippincott Williams \& Wilkins.

Bailey, R. C., Olson, J., Pepper, S. L., Porszasz, J., Barstow, T. J., \& Cooper, D. M. (1995). The level and tempo of children's physical activities: An observational study. Medicine and Science in Sports Exercise, 27, 1033-1041.

Baquet, G., Stratton, G., Van Praagh, E., \& Berthoin, S. (2007). Improving physical activity assessment in prepubertal children with high-frequency accelerometry monitoring: A methodological issue. Preventive Medicine, 44, $143-147$.

Cohen, J. (1969). Statistical power analysis for the behavioral sciences. Hillsdale, NJ: Lawrence Erlbaum.

Corder, K., Ekeland, U., Steel, R. M., Wareham, N. J., \& Brage, S. (2008). Assessment of physical activity in youth. Journal of Applied Physiology, 105, 977-987.

Department of Health, Physical Activity, Health Improvement and Prevention. (2004). At least five a week: Evidence on the impact of physical activity and its relationship to health. London: Author.

Fairclough, S., \& Stratton, G. (2006). A review of physical activity levels during elementary school physical education. Journal of Teaching in Physical Education, 25, 240-258.

Freedson, P. S., Sirard, J., Debold, N., Pate, R., Dowda, M., \& Sallis, J. (1997). Validity of two physical activity monitors in children and adolescents: Laboratory analysis of two accelerometers. In N. Armstrong, B. J. Kirby, \& J. R. Welsman (Eds.), Children and Exercise XIX (pp. 127-131). London: E \& FN Spon.

Guinhouya, C. B., \& Hubert, H. (2008). Incoherence with studies using ActiGraph MTI among children age 6-12 yr. Letter to the Editor in Chief. Medicine and Science in Sports Exercise, 40, 979.

Harrell, J. S., McMurray, R. G., Baggett, C. D., Pennell, M. L., Pearce, P. F., \& Bangdiwala, S. I. (2005). Energy costs of physical activities in children and adolescents. Medicine and Science in Sports and Exercise, 37, 329-336.

Kumahara, H., Schutz, Y., Ayabe, M., Yoshioka, M., Yoshitake, Y., Shindo, M., et al. (2004). The use of uniaxial accelerometry for the assessment of physical-activity-related energy expenditure: A validation study against whole-body indirect calorimetry. British Journal of Nutrition, 91, 235-243.

Mahar, M. T., Smith, S., Rowe, D. A., DuBose, K. D., \& McCammon, M. R. (2008). Effect of sampling interval on objectively-measured physical activity in pre-school children. Medicine and Science in Sports and Exercise, 40, S205.

McClain, J. J., Johnson, T. G., Brusseau, T. A., Jr., Washington, T. L., Tudor- Locke, C., \& Darst, P. W. (2007a). Comparison of two accelerometers for measuring physical education physical activity in 8th grade youth. Medicine and Science in Sports \& Exercise, 39, S179.

McClain, J. J., Sisson, S. B., Washington, T. L., Craig, C. L., \& Tudor- Locke, C. (2007b). Comparison of Kenz Lifecorder EX and ActiGraph accelerometers in 10-yr-old children. Medicine and Science in Sports and Exercise, 39, 630-638.

Parliamentary Office of Science and Technology. (2001). Health benefits of physical activity. Postnote number 162.

Puyau, M. R., Adolph, A. L., Vohra, F. A., Zakeri, I., \& Butte, N. F. (2004). Prediction of activity energy expenditure using accelerometers in children. Medicine and Science in Sports and Exercise, 36, 1625-1631.

Ridley, K., \& Olds, T. S. (2008). Assigning energy costs to activities in children: A review and synthesis. Medicine and Science in Sports and Exercise, 40, 1439-1446.

Sallis, J. F., \& Saelens, B. E. (2000). Assessment of physical activity by self-report: Status, limitations, and future directions. Research Quarterly for Exercise and Sport, 71(Suppl.), S1-S14.

Scruggs, P. W. (2007). Middle school physical education physical activity quantification: A pedometer steps/min guideline. Research Quarterly for Exercise and Sport, 78, 284-292.

Sherar, L., Baxter-Jones, A., Esliger, D., \& Tremblay, M. (2008). Incoherence with studies using ActiGraph MTI among children age 6-12 yr.: Response. Medicine and Science in Sports and Exercise, 40, 980.

Sirard, J. R., \& Pate, R. R. (2001). Physical activity assessment in children and adolescents. Sports Medicine, 31, $439-454$.

Strong, W. B., Malina, R. M., Blimkie, C. J. R., Daniels, S. R., Dishman, R. K., Gutin, B., et al. (2005). Evidence based physical activity for school-age youth. Journal of Pediatrics., 146, 732-737.

Treuth, M. S., Schmitz, K., Catellier, D. J., McMurray, R. G., Murray, D. M., Almeida, M. J., et al. (2004). Defining accelerometer thresholds for activity intensities in adolescent girls. Medicine and Science in Sports and Exercise, 36, 1259-1266.

Trost, S. G., Way, R., \& Okely, A. D. (2006). Predictive validity of three ActiGraph energy expenditure equations for children. Medicine and Science in Sports and Exercise, 38, 380-387. 
Tudor- Locke, C., Williams, J. L., Reis, J. P., \& Pluto, D. (2004). Utility of pedometers for assessing physical activity. Sports Medicine, 34, 281-291.

United States Department of Health and Human Services. (1996). Report of the Surgeon General: Executive summary on physical activity and health. Washington, DC: Author.

Warburton, D. E. R., Nicol, C. W., \& Bredin, S. S. D. (2006). Health benefits of physical activity: The evidence. Canadian Medical Association Journal, 174, 801-809.

Wechsler, H., Devereaux, R. S., Davis M., \& Collins, J. (2000). Using the school environment to promote physical activity and healthy eating. Preventive Medicine, 31, S121-S137.

Young, D. R., Felton, G. M., Grieser, M., Elder, J. P., Johnson, C., Lee, J. S., et al. (2007). Policies and opportunities for physical activity in middle school environments. Journal of School Health, 77, 41-47. 